\title{
Fulminant course of unilateral emphysematous pyelonephritis revealing a renal actinomycosis caused by Actinomyces meyeri, an unknown cause of septic shock
}

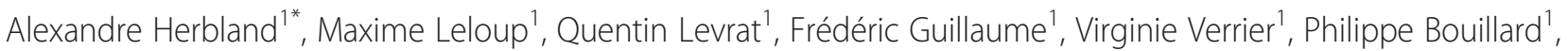
Thierry Landois ${ }^{2}$, Charlie Frédéric Ouaki ${ }^{3}$ and Olivier Lesieur ${ }^{1}$

\begin{abstract}
The objective of this case report is to describe the first case of renal actinomycosis caused by Actinomyces meyeri presenting as severe emphysematous pyelonephritis and complicated by septic shock and multi-organ failure. Emphysematous pyelonephritis is a potentially life-threatening infection mostly described in diabetic patients and predominantly caused by uropathogenic bacteria. Actinomycosis is an uncommon chronic infection due to anaerobic gram-positive bacteria that unusually involves the urinary tract. We report the first case of emphysematous pyelonephritis caused by A. meyeri in a 75-year-old non-diabetic woman. The patient presented with an altered status, fever, nausea, and vomiting lasting for 2 days. A computed tomography scan revealed unilateral emphysematous pyelonephritis. She was rapidly admitted to intensive care unit for a septic shock with multiple organ dysfunctions. A conservative management consisting in renal percutaneous drainage, supportive measures, and prolonged adapted antibiotic therapy resulted in complete recovery. This case report illustrates that renal actinomycosis should be considered in case of emphysematous pyelonephritis given the good prognosis of this infection with conservative medical treatment.
\end{abstract}

Keywords: Sepsis, Shock, Actinomyces, Actinomycosis, Emphysematous pyelonephritis, Pyuria

\section{Background}

Emphysematous pyelonephritis (EPN) is a potentially lifethreatening disease characterized by a severe necrosis of the renal parenchyma with presence of gas in the renal parenchyma, collecting system, and/or perinephric tissue. Escherichia coli is the commonest causative organism found for about $70 \%$ of cases. Other organisms like Proteus, Pseudomonas, Klebsiella, and Acinetobacter can also be found [1]. Actinomycosis is an uncommon chronic infection due to a gram-positive anaerobic bacterium. Actinomyces israelii is the most common human pathogen among all Actinomyces pathogenic species. The cervicofacial area, pelvic region, and thoracic involvement are the most common clinical presentations, but very few

\footnotetext{
* Correspondence: alexandre.herbland@orange.fr

'Service de Réanimation Polyvalente, Hôpital Saint-Louis, 17019 La Rochelle, France

Full list of author information is available at the end of the article
}

cases of renal actinomycosis (RA) are reported. Our literature review uncovered only one prior report of EPN revealing a RA after nephrectomy [2]. We report the first case of RA with Actinomyces meyeri in urine cultures presenting as an EPN complicated by a septic shock and successfully treated with conservative management.

\section{Case presentation}

A 75-year-old female presented to a local clinic with a history of fever, nausea, vomiting, and anorexia lasting for 2 days. Medical history was significant only for arterial hypertension, obesity (body mass index 29), and urinary incontinence. She was treated by oral antihypertensive therapy and solifenacin for her overactive bladder.

Physical examination revealed fever with body temperature of $101.3^{\circ} \mathrm{F}\left(38.5^{\circ} \mathrm{C}\right)$, tachypnea with respiratory rate of 25 breaths/min, normal hemodynamic parameters with blood pressure $125 / 57 \mathrm{mmHg}$, and pulse 80 beats/ 
minute. The laboratory investigations yielded an inflammatory process and moderate renal impairment. The white blood cell count was $37.6 \times 10^{9} / \mathrm{L}$ with neutrophils $85 \%$. Hemoglobin was $100.2 \mathrm{~g} / \mathrm{L}$. The platelet count was normal $263 \times 10^{9} / \mathrm{L}$, and fibrinogen was $32.89 \mu \mathrm{mol} / \mathrm{L}$. C-reactive protein was $591 \mathrm{mg} / \mathrm{L}$; the serum gammaglutamyltransferases level was increased up to $276 \mathrm{U} / \mathrm{L}$. The alkaline phosphatases level was $168 \mathrm{U} / \mathrm{L}$. The bilirubin was $9.7 \mu \mathrm{mol} / \mathrm{L}$. The urea nitrogen and creatinine serum levels were, respectively, $18 \mathrm{mmol} / \mathrm{L}$ and $282 \mu \mathrm{mol} / \mathrm{L}$. The other laboratory tests for blood chemistry panel and liver function were normal. Few minutes after her admission, the patient's blood pressure dropped dramatically with anuria and hypoxemia, so she received oxygen therapy (10 L/min with a reservoir mask) and intravenous fluid therapy with $1,500 \mathrm{~mL}$ of crystalloid (Ringer lactate) and $500 \mathrm{~mL}$ of colloid (6\% hydroxyethyl starch 130/0.4).

Urgent abdominal computed tomography (CT) scan permitted the diagnosis of EPN showing a pyonephrosis of the right kidney and presence of pneumaturia in the collecting system and in a cyst located in the lower pole of the right kidney (Figure 1). The pelvi-caliceal cavities of the right kidney appeared slightly dilated. After blood cultures, intravenous antibiotic therapy was initiated with amoxicillin/clavulanic acid $1 \mathrm{~g}$ and ofloxacin $200 \mathrm{mg}$. Despite antibiotic therapy and supportive measures, the patient's clinical condition rapidly deteriorated towards a septic shock with multiple organ dysfunction syndrome. The patient required mechanical ventilator support and intravenous vasopressor (norepinephrine). She was promptly transferred to our hospital to undergo urgent ureteral stenting.

On admission to the operating room, protective ventilation settings (controlled mandatory ventilation, tidal volume of $7 \mathrm{~mL} / \mathrm{kg}$ of predicted body weight, respiratory rate 20 breaths $/ \mathrm{min}, \mathrm{FiO}_{2}$ 1.0) resulted in a $\mathrm{PaO}_{2} /$ $\mathrm{FiO}_{2}$ ratio at 122. Hemodynamic parameters with continuous intravenous norepinephrine 0.4 gamma $/ \mathrm{kg} /$ min showed pulse 90 beats/min and mean arterial pressure $69 \mathrm{mmHg}$, and the patient was still anuric. A right retrograde cystoscopic pyelogram revealed a moderate pelvi-caliceal dilatation and also allowed a right ureteral open-ended stenting with drainage of a pyuria.

The patient was subsequently admitted to our ICU. Antibiotics were switched to intravenous ceftriaxone ( 2 g/day) plus ofloxacin (200 mg twice a day) and metronidazole (500 mg every $8 \mathrm{~h}$ ). Septic shock improved in the following 2 days. Her renal function recovered without need of renal replacement therapy and with equal diuresis of both kidneys. Despite improvement of the patient's condition, we observed a reascension of WBC at day $3\left(50 \times 10^{9} / \mathrm{L}\right)$. Gynecological examination with hysteroscopy excluded genital infection. CT scan showed a persistent posterior abscess of the right kidney with inflammatory infiltration of the perirenal space. After discussion with urologists and radiologists, a percutaneous CT scan-guided drainage was performed by a radiologist who placed a stent in the abscess and collected a hemic and purulent fluid. The patient's condition rapidly improved, so she was extubated on day 8 . On day 11 , the CT scan-guided drainage was complicated by a fistula, with the urinary tract successfully treated by a double J stenting of the right ureter.

Microscopic examination of urine collected by cystoscopy from the right pelvi-caliceal cavity showed presence of pyuria, Gram stain detected gram-positive bacilli, and culture was positive with $A$. meyeri $10^{5} \mathrm{CFU} / \mathrm{mL}$ with the use of Vitek 2 ANC card (bioMérieux, Marcy l'Etoile, France). Antibiotherapy was finally switched to amoxicillin $2 \mathrm{~g} \times 3$ per day according to the antibiogram for a duration of 3 months against $A$. meyeri. As advised by our infectious disease specialists, ofloxacin $(200 \mathrm{mg}$ twice a day) was maintained for 21 days covering a hypothetic non-diagnosed coinfection with gram-negative bacilli.

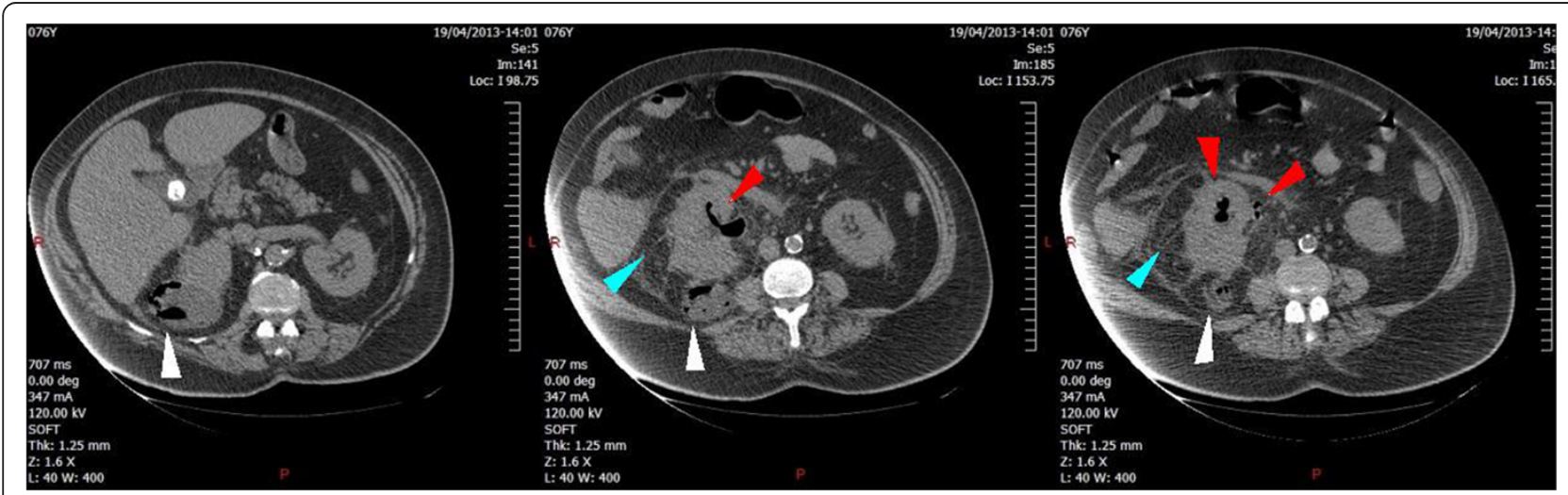

Figure 1 Emphysematous pyelonephritis (EPN) of the right kidney. Computed tomography scan demonstrates right-sided EPN with enlarged right kidney and normal left kidney. Gas is present in the renal pelvis, in the proximal ureter (red arrows), and in a posterior cyst of the right kidney (white arrows). There is also significant perirenal infiltration (blue arrows). 
Metronidazole was stopped at day 5 after identification of A. meyeri. At day 10, the patient was finally discharged from the ICU to the department of urology in stable condition. She recovered a normal renal function and was transferred back to her home at day 24. She underwent an abdominal CT scan 2 months later (Figure 2) showing regression of the right pyonephrosis with normal parenchyma, no dilatation, and persistence of a right renal cyst of reduced diameter. Four months later, ureteroscopic examination revealed no abnormality and double J stenting was retrieved.

\section{Discussion}

EPN is a renal infection caused predominantly by gramnegative rods which are capable of fermenting glucose in an anaerobic environment [3]. E. coli is the commonest organism found in about $75 \%$ of patients with EPN; other organisms like Klebsiella and Proteus are also reported [1]. EPN caused by various fungi like Candida and Aspergillus fumigatus are also sporadically documented [4-6]. EPN predominantly concerns women (female/male ratio is $6: 1$ ) and can be unilateral or bilateral $[1,7,8]$. Around $95 \%$ of patients developing EPN have uncontrolled diabetes mellitus (DM), but other associated factors are reported as drug abuse, neurogenic bladder, alcoholism, immune-compromised status, anatomical anomaly, or presence of urinary tract obstruction [1,7-10]. In our case, DM was not present, but the patient had two associated risks for EPN: urinary incontinence and a cyst on the right kidney, an anatomical particularity which may have induced local urine retention with moderate obstruction of the pelvi-caliceal cavities.

Diagnosis was made by CT scan which is currently the radiographic method of choice both for diagnosing EPN and demonstrating the extent of the disease [1]. Sonographic diagnosis of EPN is also possible by the recognition of echogenic foci with 'dirty' shadowing in a non-dependent position, highly suggestive of gas inside the kidney [11]. Ultrasonography can also be useful to realize guided percutaneous drainage of renal abscesses. But the presence of gas within the renal parenchyma in EPN produces artifactual reverberation echoes, compromising the quality of the ultrasound images. Thus, we opted for CT scan-guided percutaneous drainage of the renal abscess. Moreover, CT scan allows much more accuracy than ultrasonography for the drain placement because the entire guidewire or drainage catheter is usually demonstrated more clearly by CT than by ultrasound. In patients with EPN, beside aggressive medical management (prompt appropriate antibiotics and resuscitation measures with multi-organ support), percutaneous drainage seems to be the most successful management compared with medical management alone or plus nephrectomy with the lowest mortality at $13.5 \%(P<0.001)[12]$.

Actinomycosis is a rare chronic infection due to anaerobic gram-positive bacteria that unlikely involves the urinary tract [13-15]. This infection is due to a group of bacteria which are normal commensals found in the mouth and gastrointestinal tract, with $A$. israelii being the most common agent identified in human infections and particularly in RA. It seems that Actinomyces are able to invade mucous membranes under certain circumstances such as mucosal alteration secondary to trauma or infection by other organisms [16]. Poor hygiene, underlying diseases, or immunosuppression can be found in patients with actinomycosis, but predisposing factors are not systematically documented [17]. In our case, it is still unclear how the patient contracted the infection, as there was neither history of previous surgical treatment nor other risk factors identified. Hematogenous origin can be hypothesized without certainty.

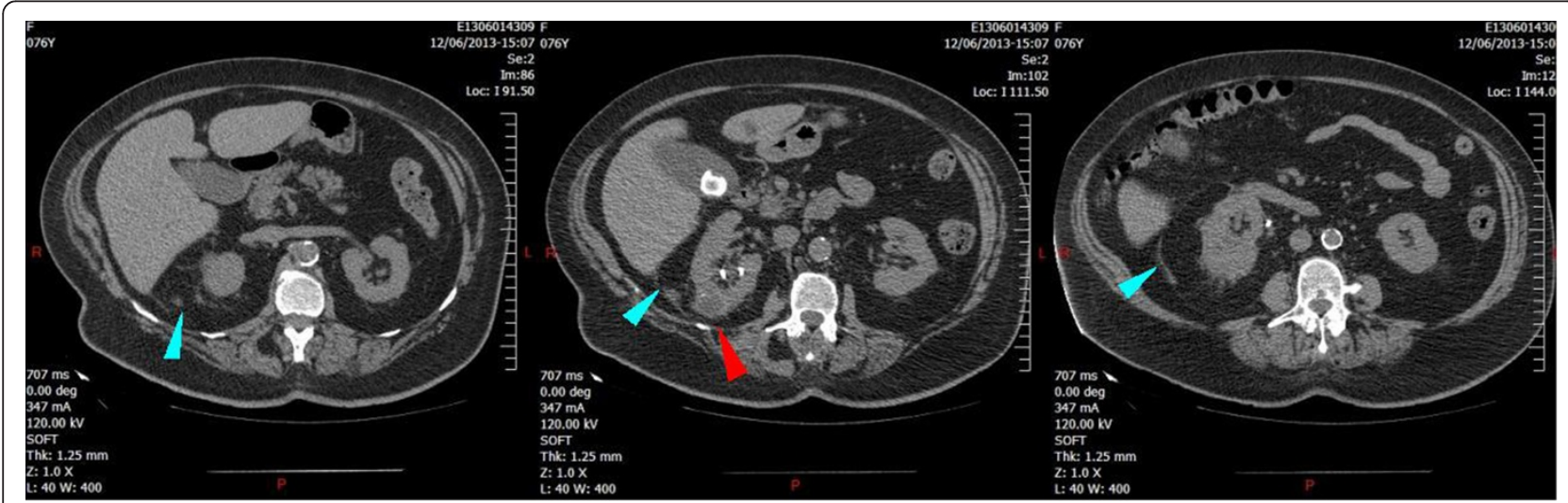

Figure 2 The 2-month follow-up computed tomography scan. The follow-up computed tomography scan shows the normalizing size and complete absence of gas in the right kidney. A small posterior cyst of the right kidney is still present (red arrow). Perirenal infiltration has almost disappeared (blue arrows). 
Actinomyces meyeri was first isolated from a patient with a lung empyema by Meyer in 1911, who termed it Streptothrix. The nomenclature was changed to Actinobacterium meyerii by Prevot in 1956 and subsequently to Actinomyces meyeri by Holderman in 1977 [18]. A recent review of the literature revealed only 32 documented cases of infection with A. meyeri [19]. According to this article, 13 patients had a disseminated disease, and the most frequent sites of infection were pulmonary, gastrointestinal, skin, and soft tissues, respectively. No case of urinary tract infection was reported.

The few cases of RA published are characterized by a medical presentation mimicking malignancy with pseudotumoral aspect, insidious course, and non-specific symptoms [20-22]. The diagnosis is difficult, and only few patients have avoided nephrectomy or at least surgical exploration. Diagnosis can be made by ultrasound or CT-guided fine needle biopsy $[23,24]$. In our particular case, microbiological diagnosis of actinomycosis was based on concordant Gram stain result (gram-positive bacilli) and positive urine culture collected in the upper urinary tract under ureteroscopy. As we opted for a non-invasive treatment, we did not perform any biopsy for histological examination. Furthermore, other urine and blood cultures (including those taken before first antibiotherapy) failed to grow any other microorganism.

A case of RA presenting as EPN and complicated by septic shock was already reported from Taiwan [2]. The patient recovered after medical treatment and nephrectomy. Diagnostic of RA was made on histological examination of the surgical kidney and ureteral specimens. Microbiological identification of Actinomyces species is not detailed in the article, and the patient had a urinary tract coinfection with E. coli that could explain such an emphysematous presentation. Our search for published literature did not reveal any report of urinary tract infection or EPN caused by $A$. meyeri.

Actinomyces are usually susceptible to a wide variety of antimicrobial agents including penicillin G, cephalosporins, tetracycline, erythromycin, clindamycin, imipenem, and streptomycin $[25,26]$ but are resistant to fluoroquinolones which are widely prescribed for urinary tract infections. There is no consensus recommendation for medical treatment of RA and no particular sensitivity or resistance to antibiotics identified for $A$. meyeri given the small number of cases reported [19]. Clinical experience supports the use of intravenous penicillin $G$ as the drug of choice (18 to 24 million U/day) for 2 to 6 weeks followed by oral therapy. For other Actinomyces infections, a prolonged duration of antibiotic therapy is advised to avoid relapse varying to 2 months for the localized disease to 12 months for more extensive and disseminated disease [26]. The treatment needs to be individualized based on the clinical and radiological response.

\section{Conclusions}

RA is a rare and difficult infection to recognize and should be considered of etiological diagnosis for EPN. This first case report of severe EPN secondary to RA caused by $A$. meyeri clearly illustrates that EPN is a potentially life-threatening disease. A CT scan-guided percutaneous drainage of renal abscess was a safe and effective procedure which permitted decisive improvement of the patient's condition. Awareness of this entity may encourage physicians to make the diagnosis and to implement effective long-lasting antibiotic therapy, avoiding futile surgery and preserving further renal function.

\section{Consent}

Written informed consent was obtained from the patient for publication of this case report and any accompanying images. A copy of the written consent is available for review by the Editor-in-Chief of this journal.

\section{Competing interests}

The authors declare that they have no competing interests.

\section{Authors' contributions}

$\mathrm{AH}, \mathrm{ML}, \mathrm{FG}, \mathrm{QL}, \mathrm{W}, \mathrm{PB}$, and $\mathrm{OL}$ participated to the management of the patient in the ICU. TL interpreted the TDM and performed the renal-guided biopsy. CFO was the urologist in charge of the patient. All authors read and approved the final manuscript.

\section{Author details}

'Service de Réanimation Polyvalente, Hôpital Saint-Louis, 17019 La Rochelle, France. ${ }^{2}$ Service de Radiologie, Hôpital Saint-Louis, 17019 La Rochelle, France. ${ }^{3}$ Service d'Urologie, Hôpital Saint-Louis, 17019 La Rochelle, France.

Received: 13 January 2014 Accepted: 12 June 2014

Published: 8 July 2014

\section{References}

1. Ubee SS, McGlynn L, Fordham M: Emphysematous pyelonephritis. BJU Int 2011, 107:1474-1478.

2. Lin $\mathrm{Y}-\mathrm{H}$, Chen $\mathrm{H}-\mathrm{W}$, Chuang $\mathrm{C}-\mathrm{K}, \mathrm{Ng} \mathrm{K}-\mathrm{F}$, Wang $\mathrm{H}-\mathrm{H}$ : Renal actinomycosis presented as emphysematous pyelonephritis: a case report. J Taiwan Urol Assoc 2009, 20:181-183.

3. Huang JJ, Tseng CC: Emphysematous pyelonephritis: clinicoradiological classification, management, prognosis, and pathogenesis. Arch Intern Med 2000, 160:797-805.

4. Hildebrand TS, Nibbe L, Frei U, Schindler R: Bilateral emphysematous pyelonephritis caused by Candida infection. Am J Kidney Dis 1999, 33:E10.

5. Ahmad M, Dakshinamurty KV: Emphysematous renal tract disease due to aspergillus fumigatus. J Assoc Physicians India 2004, 52:495-497.

6. Kamaliah MD, Bhajan MA, Dzarr GAA: Emphysematous pyelonephritis caused by Candida infection. Southeast Asian J Trop Med Public Health 2005, 36:725-727.

7. Shokeir AA, El-Azab M, Mohsen T, El-Diasty T: Emphysematous pyelonephritis: a 15-year experience with 20 cases. Urology 1997, 49:343-346.

8. Tahir H, Thomas G, Sheerin N, Bettington H, Pattison JM, Goldsmith DJ: Successful medical treatment of acute bilateral emphysematous pyelonephritis. Am J Kidney Dis 2000, 36:1267-1270.

9. Tseng C-C, Wu J-J, Wang M-C, Hor L-I, Ko Y-H, Huang J-J: Host and bacterial virulence factors predisposing to emphysematous pyelonephritis. Am J Kidney Dis 2005, 46:432-439.

10. Schmidt S, Foert E, Zidek W, van der Giet M, Westhoff TH: Emphysematous pyelonephritis in a kidney allograft. Am J Kidney Dis 2009, 53:895-897. 
11. Hui SY, Cheung CW, Hui KT, She HL: Sonographic diagnosis of emphysematous pyelonephritis in a clinically stable patient. Hong Kong Med J 2010, 16:319.

12. Somani BK, Nabi G, Thorpe P, Hussey J, Cook J, N'Dow J: Is percutaneous drainage the new gold standard in the management of emphysematous pyelonephritis? Evidence from a systematic review. J Urol 2008, 179:1844-1849.

13. Chang D-S, Jang WI, Jung JY, Chung S, Choi DE, Na K-R, Lee KW, Shin Y-T: Renal actinomycosis with concomitant renal vein thrombosis. Clin Nephrol 2012, 77:156-160.

14. Kanemiya T, Arai H, Murosaki N, Honda M, Osaki T, Hara H, Yoshida K: Renal actinomycosis with pneumonia. Hinyokika Kiyo 2012, 58:155-158.

15. Mallick S, Klein JF: Renal actinomycosis with fistulized lumbar abscess. Prog Urol 2000, 10:587-589.

16. Garner JP, Macdonald M, Kumar PK: Abdominal actinomycosis. Int I Surg Lond Engl 2007, 5:441-448.

17. Acevedo F, Baudrand R, Letelier LM, Gaete P: Actinomycosis: a great pretender: case reports of unusual presentations and a review of the literature. Int J Infect Dis 2008, 12:358-362.

18. Apothéloz C, Regamey C: Disseminated infection due to Actinomyces meyeri: case report and review. Clin Infect Dis 1996, 22:621-625.

19. Fazili T, Blair D, Riddell S, Kiska D, Nagra S: Actinomyces meyeri infection: case report and review of the literature. J Infect 2012, 65:357-361.

20. Dusaud M, Durand X, Salin A, Houlgatte A: Renal pseudotumoral actinomycosis: a case report. Prog Urol 2011, 21:580-582.

21. Dieckmann KP, Henke RP, Ovenbeck R: Renal actinomycosis mimicking renal carcinoma. Eur Urol 2001, 39:357-359.

22. Horino T, Yamamoto M, Morita M, Takao T, Yamamoto Y, Geshi T: Renal actinomycosis mimicking renal tumor: case report. South Med J 2004, 97:316-318

23. Hyldgaard-Jensen J, Sandstrøm HR, Pedersen JF: Ultrasound diagnosis and guided biopsy in renal actinomycosis. Br J Radiol 1999, 72:510-512.

24. Dhanani NN, Jones DM, Grossman HB: Medical management of renal actinomycosis. J Urol 2004, 171:2373-2374.

25. Khalaff H, Srigley JR, Klotz LH: Recognition of renal actinomycosis: nephrectomy can be avoided: report of a case. Can I Surg 1995, 38:77-79.

26. Kim SR, Jung LY, Oh I-J, Kim Y-C, Shin K-C, Lee MK, Yang S-H, Park HS, Kim M-K, Kwak JY, Um S-J, Ra SW, Kim WJ, Kim S, Choi E-G, Lee YC: Pulmonary actinomycosis during the first decade of 21st century: cases of 94 patients. BMC Infect Dis 2013, 13:216.

doi:10.1186/2052-0492-2-42

Cite this article as: Herbland et al.: Fulminant course of unilateral emphysematous pyelonephritis revealing a renal actinomycosis caused by Actinomyces meyeri, an unknown cause of septic shock. Journal of Intensive Care 2014 2:42.

\section{Submit your next manuscript to BioMed Central and take full advantage of:}

- Convenient online submission

- Thorough peer review

- No space constraints or color figure charges

- Immediate publication on acceptance

- Inclusion in PubMed, CAS, Scopus and Google Scholar

- Research which is freely available for redistribution 